\title{
Percepção de preconceito e autoestima entre adolescentes em contexto familiar e em situação de acolhimento institucional
}

\section{Perception of prejudice and self-esteem among adolescents in a familiar context and in out-of-home care context}

\section{Anelise Lopes Rodrigues*}

Universidade Federal do Rio Grande do Sul - UFRGS, Porto Alegre, Rio Grande do Sul, Brasil

\section{Lara Lages Gava**}

Universidade Federal do Rio Grande do Sul - UFRGS, Porto Alegre, Rio Grande do Sul, Brasil

\section{J orge Castellá Sarriera***}

Universidade Federal do Rio Grande do Sul - UFRGS, Porto Alegre, Rio Grande do Sul, Brasil

\section{Débora Dalbosco Dell'Aglio****}

Universidade Federal do Rio Grande do Sul - UFRGS, Porto Alegre, Rio Grande do Sul, Brasil

\begin{abstract}
RESUMO
Este estudo investigou as relações entre a percepção de preconceito e a autoestima em um grupo de 556 adolescentes escolares, que vivem com suas famílias, comparativamente a um grupo de 96 adolescentes em situação de acolhimento institucional. Foi utilizado o Questionário da Juventude Brasileira para avaliar questões referentes à percepção de preconceito sofrido e autoestima. A análise univariada de variância revelou diferenças significativas entre os grupos na variável preconceito e entre os sexos na variável autoestima, sendo que o grupo de adolescentes acolhidos apresentou maior percepção de preconceito e as participantes do sexo feminino menor autoestima em ambos os grupos. Conclui-se que, embora os participantes institucionalizados tenham maior percepção de preconceito, isso não se reflete em médias significativamente mais baixas de autoestima, indicando que as instituições de acolhimento podem representar um espaço de proteção capaz de fornecer, ainda que provisoriamente, condições para um desenvolvimento saudável.
\end{abstract}

Palavras-chave: preconceito, autoestima, adolescentes, institucionalização.

\section{ABSTRACT}

This paper studies the relation between perception of prejudice and selfesteem in a group of 556 adolescent students, living with their families, compared to a group of 96 adolescents living in out-of-home care. The 
Brazilian Youth Questionnaire was employed, in which questions about perception of prejudice and self-esteem were asked to both groups. Univariate Analyses of Variance indicated significant differences between the two groups with respect to prejudice, as well as between genders with regards to self-esteem. The out-of-home care group showed a greater prejudice perception and the female participants in both groups showed lower self-esteem. It can be concluded that, although institutionalization may contribute to a greater perception of prejudice, this did not reflect in a significantly lower mean for self-esteem. This result indicates that these institutions may represent a place capable of, even temporarily, providing protection and conditions for a healthy development.

Keywords: prejudice, self-esteem, adolescents, institutionalization.

\section{Percepção de preconceito e autoestima}

Em diferentes sociedades, ao longo da história da humanidade, determinados grupos têm sido alvo de preconceitos. Evidências baseadas em pesquisas apontam que as pessoas possuem, geralmente, preconceito e estereótipos negativos sobre diversos grupos tais como negros, mulheres, pessoas portadoras de deficiência física, obesos, doentes mentais, homossexuais, pessoas pobres, dentre outros (Crocker \& Major, 1989; Major \& O'brien, 2005; Sidanius \& Pratto, 1999).

Gordon Allport (1954/1979), em sua obra clássica intitulada The nature of prejudice, afirmou que o homem não nasce preconceituoso, sendo o preconceito algo aprendido e que, na medida em que nega a dignidade humana, é responsável por promover uma ruptura na unidade fundamental entre as pessoas. Allport define o preconceito como sendo

(...) um sentimento ou atitude hostil direcionada para uma pessoa só porque ele ou ela pertence a um grupo ao qual se atribuiu qualidades questionáveis. É uma antipatia baseada em generalização falha e inflexível ou estereótipos (...) que pode ser sentida ou expressa, e é dirigida para um grupo como um todo ou para um indivíduo porque ele ou ela é um membro desse grupo (Allport, 1979, p. 1).

Já o estigma ou estigmatização ocorre quando uma pessoa possui (ou acredita-se que possui) "algum atributo ou característica que lhe transmite uma identidade social que é desvalorizada em um contexto social específico" (Crocker \& Major, 1989, p. 505). Observa-se, assim, que o estigma não reside na pessoa isoladamente, mas sim na pessoa em um determinado contexto social, seja por sua aparência física, seu comportamento ou pertencimento a um grupo (Major \& O'brien, 2005). Para alguns autores, os estigmas decorrem de preconceitos e ao mesmo tempo os alimentam, cristalizando 
pensamentos e expectativas com relação a indivíduos e grupos (Clam, 2009). Em suma, em termos didáticos, pode-se conceituar o preconceito como qualquer atitude negativa em relação a uma pessoa ou a um grupo social que derive de uma ideia preconcebida sobre tal pessoa ou grupo; já o estigma refere-se a uma "marca ou rótulo atribuídos a pessoas e grupos, sempre associado a uma forma de simplificação, de desqualificação dessa pessoa e/ou grupo"; a discriminação, por sua vez, diz respeito à ação de discriminar, tratar de forma diferente, anular, tornar invisível, excluir, marginalizar (Clam, 2009, p. 35). Observa-se que a aparente confusão existente na literatura acerca desses termos deve-se ao fato de que o preconceito, o estigma e a discriminação são processos que se encontram, de certa forma, interligados.

Historicamente, a psicologia tem desenvolvido pesquisas que investigam as causas de preconceitos, discriminações e estereótipos (Major \& O’brien, 2005). Entretanto, apenas recentemente estudos têm se concentrado em compreender os efeitos psicológicos desses processos. Nos últimos anos, evidencia-se uma série de estudos de caráter teórico e empírico que - no intuito de suprir essa lacuna buscam determinar os efeitos do preconceito e da discriminação sobre a autoestima em diferentes populações (Bianchi, Zea, Belgrave, \& Echverry, 2002; Bourguignon, Seron, Yzerbyt, \& Herman, 2006; Crocker \& Major, 1989; Crocker \& Quinn, 2000).

A autoestima tem sido reconhecida, nas culturas ocidentais, como um aspecto central do funcionamento psicológico, sendo fortemente relacionada com a qualidade afetiva da experiência, de modo que pessoas com autoestima elevada relatam mais afeto positivo, mais satisfação de vida, menos desesperança e menos depressão, quando comparadas a pessoas com baixa autoestima (Crocker \& Quinn, 2000). Diener (1984) elegeu a autoestima como o mais forte preditor de satisfação geral com a vida, na população norte-americana, sendo considerado, à época, mais forte inclusive que resultados demográficos e objetivos, tais como idade, renda, escolaridade e estado civil, além de outras variáveis psicológicas.

Conceitualmente, a autoestima foi definida por Rosenberg (1965) como a avaliação do indivíduo sobre seu próprio valor e adequação, o que se reflete em sentimentos, pensamentos e atitudes de aprovação ou desaprovação de si. Em relação às características pessoais, ou individuais, é considerada como um dos construtos que formam o núcleo periférico do bem-estar, junto à percepção de controle, o apoio social percebido, o otimismo vital, entre outros (Casas, 2010).

Em um estudo extensivo acerca das relações entre estigma social e autoestima, Crocker e Major (1989) apontaram que várias teorias psicológicas, consideradas clássicas, previram que grupos estigmatizados apresentavam a tendência de internalizar a visão negativa a eles atribuída pela sociedade. Assim, partiam do pressuposto de que tais grupos deviam possuir menor autoestima do 
que membros de grupos não estigmatizados. No entanto, resultados de estudos empíricos empreendidos à época não corroboraram tal previsão. Um estudo de meta-análise, realizado por Twenge e Crocker (2002), investigou medidas de autoestima e diferenças raciais. De acordo com os resultados obtidos, o grupo de afroamericanos apresentou maior autoestima comparado ao grupo de americanos brancos, não apoiando a previsão clássica. Para Crocker e Major (1989), tal discrepância entre a teoria clássica e dados empíricos sobre as consequências do estigma social para a autoestima pode ser explicada considerando-se as diferenças individuais, situacionais e, mais especificamente, as diferentes formas a partir das quais os membros de grupos estigmatizados podem adotar mecanismos visando a proteger seu autoconceito. Esses autores propõem, assim, um modelo no qual o processo de estigma social, paradoxalmente, apresenta uma propriedade autoprotetiva, a partir da existência de um repertório de mecanismos que, quando adotados, teriam a capacidade de proteger a autoestima dos membros de grupos estigmatizados ou vítimas de preconceito. As principais medidas autoprotetivas consistiriam em: (a) atribuir o preconceito ou estigma negativo contra seu grupo e não contra si próprio; (b) comparar seus resultados e desempenhos com pessoas pertencentes a seu próprio grupo, ao invés de com grupos não estigmatizados e relativamente favorecidos; e (c) diante de tarefas, seletivamente, desvalorizar as dimensões em que seu grupo vai mal e valorizar as dimensões em que seu grupo se destaca.

Também Bourguignon, Seron, Yzerbyt e Herman (2006) investigaram a influência da percepção de discriminação pessoal e da discriminação voltada para o grupo. No primeiro estudo, realizado com um grupo de imigrantes africanos, foi verificado que a discriminação pessoal relacionou-se negativamente com a autoestima, enquanto que a discriminação voltada para o grupo foi associada positivamente com essa variável, confirmando a hipótese inicial de que a identificação grupal serviu como um amortecedor entre a discriminação pessoal e autoestima. Replicando o estudo para um grupo de mulheres, foi possível observar que a percepção de discriminação grupal pode ser relacionada positivamente com a autoestima, pelo fato de que as pessoas sentem-se menos sós na sua situação, aliviando assim os maus efeitos gerados pela exclusão.

Major, Quinton e McCoy (2002), a partir de uma série de pesquisas sobre as implicações psicológicas do preconceito e discriminação percebidos, passaram a adotar um modelo de estresse e coping para entender as respostas dos alvos de preconceito e discriminação. Em direção semelhante, Major, McCoy, Kaiser e Quinton (2003) relacionam dois modelos teóricos que, tradicionalmente, têm buscado explicar como o preconceito afeta a autoestima de seus alvos. Um primeiro modelo, denominado de estímulo-resposta, assume que o 
preconceito tem um efeito direto negativo sobre a autoestima. $O$ segundo, chamado de estímulo-percepção-resposta, reconhece que a percepção que 0 sujeito tem do preconceito não se reflete diretamente em prejuízo, porém prevê que a percepção subjetiva de ser um alvo de prejuízo tem um efeito negativo direto sobre a autoestima. Ao considerar, no entanto, que esses modelos têm se mostrado inadequados perante a complexidade do problema, os autores do estudo propõem a adoção de um terceiro modelo, denominado modelo transacional. De acordo com esse modelo, que leva em consideração os fatores pessoais, situacionais e estruturais, parte-se da noção de que sujeitos que são alvos de preconceito não respondem de modo uniforme e, assim sendo, a autoestima e demais respostas emocionais ao preconceito são determinadas por avaliações cognitivas relacionadas ao prejuízo, bem como por estratégias de enfrentamento utilizadas em resposta a esses eventos.

Também Pascoe e Richman (2009), em estudo de meta-análise envolvendo 134 publicações sobre a temática, forneceram uma descrição abrangente das relações entre as múltiplas formas de discriminação percebidas e seus diversos tipos de efeito na saúde. Foi evidenciado que a discriminação percebida tem um efeito negativo significativo sobre a saúde física e mental, aumentando as respostas ao estresse e relacionando-se à baixa adesão a condutas saudáveis. Os autores relatam que o apoio social, estilos de enfrentamento ativo e identificação grupal apresentaram uma função protetora frente à percepção de discriminação.

\section{Institucionalização e preconceito}

No Brasil, observa-se que as instituições de acolhimento têm sido marcadas por estigmas negativos. Por consequência, o preconceito ganha força contra crianças e adolescentes, em situação de acolhimento, bem como a discriminação e exclusão social a que esses são constantemente submetidos (Buffa, Teixeira, \& Rosseti-Ferreira, 2010). A promulgação do Estatuto da Criança e do Adolescente (ECA) em 1990, resultante de mobilizações sociais pela integridade dos direitos da criança e do adolescente (Ayres, Coutinho, Sá, \& Albernaz, 2010), trouxe mudanças significativas em relação à institucionalização, uma vez que diferenciou os adolescentes considerados vítimas, daqueles considerados infratores (Buffa et al., 2010). Viabilizou-se, assim, a formulação de propostas de intervenção pertinentes a cada um destes grupos e a proposição de uma nova política de acolhimento ao grupo dos adolescentes vitimizados, com significativa alteração na atenção despendida a esses, contrária à prática de segregação social e confinamento (Rizzini, 2006). O Estatuto da Criança e do Adolescente [ECA] (1990) previu ainda, em seu artigo 101, que o acolhimento da criança ou do 
adolescente em situação de vulnerabilidade deve ser considerado medida última, de caráter provisório e excepcional, consideradas todas as possibilidades de permanecer com os seus familiares. A partir do estabelecimento do ECA ocorreu um reordenamento do sistema de abrigos no Brasil (Silva, 2004), com mudanças positivas quanto à qualidade da atenção. Houve alterações relativas ao tamanho dos abrigos (os quais passaram a ser configurados em unidades pequenas, com poucos integrantes), manutenção de uma atenção mais personalizada, maior participação em atividades comunitárias, preservação do grupo de irmãos sem separação por sexo, redução do tempo de permanência nos abrigos, e manutenção do vínculo familiar, entre outras.

As recomendações da lei 8.069/90 (ECA, 1990) foram ainda aperfeiçoadas na Lei no 12.010 (2009), sancionada em agosto de 2009, que alterou a sistemática prevista para garantia do direito à convivência familiar a todas as crianças e adolescentes, adotando a nomenclatura de "acolhimento institucional" e definindo a permanência de no máximo dois anos em instituição, além de determinar avaliações a cada seis meses. Essa lei trouxe avanços em relação ao que já era previsto pelo ECA, embora a situação nas instituições ainda não esteja apresentando muitas mudanças, tendo em vista a dificuldade em sua execução.

Entre os direitos previstos pelo ECA (1990), destaca-se o direito à convivência familiar e comunitária. $O$ abrigo ou instituição de acolhimento deve ser concebido como espaço de proteção e proporcionar características residenciais, possibilitando a oportunidade de participar na vida da comunidade mediante a utilização de bens e recursos disponíveis, como escolas, áreas de lazer, centros médicos, quadras esportivas, dentre outras (Nunes, Santos, Martins, \& Monteiro, 2010). Deve ser, portanto, um espaço que forneça condições para o saudável desenvolvimento do sujeito ali provisoriamente residente.

Segundo o Instituto de Pesquisa Econômica Aplicada (Silva, 2004), em 2003, cerca de 80.000 crianças e adolescentes viviam em instituições de acolhimento no Brasil. Apesar das previsões legais, estudos apontam que muitas instituições ainda apresentam modos de funcionamento inadequados e prejudiciais. A não preservação da individualidade e $o$ uso de práticas disciplinares exercidas pelo controle coercitivo (Prada, Williams, \& Weber, 2007), além das falhas de reinserção no contexto familiar, são alguns exemplos de práticas que podem prejudicar o desenvolvimento das crianças atendidas (Buffa et al., 2010; Siqueira, Zoltowski, Giordani, Otero, \& Dell' Aglio, 2010).

Por outro lado, Arpini (2003) aponta a necessidade de se reconsiderar a perspectiva institucional, problematizando a concepção social de que instituições são alternativas fracassadas e compreendendo que, 
em muitos casos, elas se apresentam como uma alternativa de acolhimento. Isso possibilita que crianças e adolescentes sejam retirados de situações de risco, abandono ou violência às quais eram submetidos, oferecendo-Ihes um local de maior tranquilidade e apoio até que suas vidas sejam reestruturadas. A autora lembra ainda que a institucionalização é resultante, muitas vezes, de situações de violência e abandono praticados pelas próprias famílias. Estar em um abrigo, portanto, constitui-se em um forte estigma social, uma vez que muitas pessoas ainda julgam que a criança ou o adolescente possui algum sério problema em seu histórico, caso contrário não estaria institucionalizado.

A situação de acolhimento institucional confere aos adolescentes, portanto, uma identidade social que potencialmente marca-os como "diferentes" perante a sociedade, podendo torná-los alvo de preconceito, exclusão e estigmatizações. Considerando pesquisas que apontam a influência da percepção de preconceito na autoestima de grupos estigmatizados, esse trabalho buscou investigar as relações entre a autoestima e a percepção de preconceito em um grupo de adolescentes em situação de acolhimento institucional, comparativamente a um grupo de adolescentes que vivem com suas famílias.

\section{Método}

\subsection{Participantes}

Participaram deste estudo 652 adolescentes, de ambos os sexos, com idade entre 12 e 17 anos (Média=14,88; $\mathrm{DP}=1,35$ ), sendo 400 do sexo feminino $(61,3 \%)$ e 252 do sexo masculino $(38,7 \%)$. Os participantes deste estudo estavam inseridos nos seguintes contextos: Grupo 1 (G1), 556 adolescentes (342 do sexo feminino) que estudavam entre a $6^{\text {a }}$ série do Ensino Fundamental e o $3^{\circ}$ ano do Ensino Médio, em escolas públicas da cidade de Porto Alegre/RS e que viviam com suas famílias; Grupo 2 (G2), 96 adolescentes (58 do sexo feminino) igualmente estudantes de escolas públicas e que estavam sob proteção em instituições governamentais de acolhimento, municipais (Fundação de Assistência Social e CidadaniaFASC), estaduais (Fundação de Proteção Especial) e não governamentais (ONGs conveniadas com a prefeitura de Porto Alegre e cidades vizinhas). Cabe salientar que a proporção referente ao sexo e idade se manteve nos dois grupos.

\subsection{I nstrumento}


Os participantes responderam ao Questionário da Juventude Brasileira (Dell Aglio, Koller, Cerqueira-Santos, \& Colaço, 2011), instrumento composto de 77 questões que investigam fatores de risco e proteção no desenvolvimento. Para este estudo foram consideradas as variáveis sociodemográficas e foram observadas as questões referentes à autoestima (questão 74) e à percepção de preconceito (questão 65) para ambos os grupos.

A questão 74, do Questionário da Juventude Brasileira, foi constituída pelos itens da Escala de Autoestima de Rosenberg (1965), baseada na adaptação de Hutz e Zanon (2011). Esta escala avalia a autoestima através de dez itens fechados que consideram aspectos positivos (ex.: "Eu tenho motivos para me orgulhar na vida") e negativos (ex.: "Eu sinto vergonha de ser do jeito que sou") do autovalor. As questões estão dispostas no formato Likert de cinco pontos, variando entre "Nunca" e "Sempre". Com relação à pontuação, quanto maior o escore obtido na escala, maior o nível de autoestima do indivíduo.

A questão 65, por sua vez, foi construída com base na literatura e consiste em uma escala de 12 itens fechados que avalia a percepção de preconceito em diferentes aspectos, tais como local de moradia, sexo, cor da pele, religião, nível socioeconômico, escolhas sexuais e aparência física, dentre outros. As questões estão dispostas no formato Likert de cinco pontos, variando entre "Nunca" e "Sempre". $\mathrm{Na}$ medida em que não há itens negativos, a pontuação final foi obtida a partir da soma dos itens da escala, de modo que, quanto maior o escore obtido, maior a percepção de preconceito sofrida pelos adolescentes.

\section{Procedimentos e considerações éticas}

Os aspectos éticos, que garantem a integridade dos participantes, foram assegurados, tendo como base a Resolução no 196 (Ministério da Saúde, 2002). O estudo foi aprovado pelo Comitê de Ética em Pesquisa do Instituto de Psicologia da UFRGS, sob o protocolo número 2009060. Foi solicitada a assinatura do Termo de Consentimento Livre e Esclarecido aos pais e às instituições. Especificamente para a composição dos participantes do Grupo 1 (adolescentes que viviam com suas famílias) foi constituída uma amostra aleatória por conglomerados através de sorteio das escolas públicas municipais e estaduais da cidade de Porto Alegre e, posteriormente, sorteio das turmas de adolescentes em cada escola selecionada. O número de participantes foi obtido através do cálculo amostral, a partir do número total de alunos de ensino fundamental e ensino médio matriculados em escolas públicas de Porto Alegre, com uma margem de erro estabelecida de $4 \%$ (Barbetta, 2001). Para a 
composição do Grupo 2, foram considerados critérios de inclusão na amostra: adolescentes que apresentavam condições cognitivas para responder ao instrumento da pesquisa e que estivessem há pelo menos 30 dias em acolhimento institucional. Todos os adolescentes que estavam em acolhimento nas instituições contatadas, durante 0 período de coleta de dados, foram convidados a participar. Dessa forma, a amostragem foi não probabilística, mas representa os adolescentes nesta situação, tendo em vista que a amostra obtida representa $59 \%$ do total de adolescentes que possuíam os critérios estipulados na rede de acolhimento governamental e não governamental conveniada durante o período de coleta.

Os adolescentes de ambos os grupos foram convidados a participar do estudo, esclarecendo a voluntariedade da participação, a garantia de sigilo das informações pessoais e a possibilidade de desistência a qualquer momento. Os adolescentes que concordaram em participar do estudo também assinaram o Termo de Consentimento Livre e Esclarecido. $O$ instrumento foi aplicado nas próprias escolas e instituições de acolhimento, com duração de aproximadamente 60 minutos. Foi disponibilizada a assistência por parte da equipe de pesquisa, que foi capacitada para este tipo de coleta de dados, no caso de participantes que necessitaram de apoio durante ou imediatamente após a realização da coleta de dados.

\section{Análise dos dados}

Foram realizadas análises descritivas e teste univariados (ANOVA), considerando as variáveis dependentes "autoestima" e "percepção de preconceito" e as variáveis independentes "grupo", "idade" e "sexo" dos participantes que compuseram a amostra.

\section{Resultados e discussão}

Na Tabela 1, são apresentados os dados descritivos, as médias e desvios padrão da amostra para as variáveis percepção de preconceito e autoestima, por grupo e sexo. 
Anelise Lopes Rodrigues, Lara Lages Gava, Jorge Castellá Sarriera, Débora Dalbosco Dell'Aglio Percepção de preconceito e autoestima entre adolescentes em contexto familiar e em situação de acolhimento institucional

Tabela 1

Dados Descritivos, Médias e Desvios Padrão da Amostra para as Escalas por Grupo e Sexo

\begin{tabular}{|c|c|c|c|c|c|c|}
\hline & \multicolumn{3}{|c|}{ G1 (família) } & \multicolumn{3}{|c|}{ G2 (acolhimento institucional) } \\
\hline & $\begin{array}{l}\text { Masculino } \\
(n=214)\end{array}$ & $\begin{array}{l}\text { Feminino } \\
(n=342)\end{array}$ & $\begin{array}{c}\text { Total } \\
(n=556)\end{array}$ & $\begin{array}{c}\text { Masculino } \\
(n=38)\end{array}$ & $\begin{array}{c}\text { Feminino } \\
(n=58)\end{array}$ & $\begin{array}{c}\text { Total } \\
(n=96)\end{array}$ \\
\hline Preconceito & $15,46(4,95)$ & $15,31(4,06)$ & $15,37(4,42)$ & $17,24(7,42)$ & $18,12(6,34)$ & $17,77(6,76)$ \\
\hline Autoestima & $42,31(6,97)$ & $41,11(7,34)$ & $41,57(7,21)$ & $43,15(5,71)$ & $38,03(7,88)$ & $40,06(7,50)$ \\
\hline
\end{tabular}

No que se refere à percepção de preconceito, pode-se observar a partir da Tabela 1 que as médias dos adolescentes em situação de acolhimento institucional (G2) se mostraram mais elevadas quando comparadas às médias dos adolescentes que viviam com suas famílias (G1), em ambos os sexos.

Observa-se ainda, no tocante à variável autoestima, que a média dos adolescentes em contexto familiar se mostrou levemente mais alta quando comparadas ao grupo de adolescentes institucionalizados. Entretanto, levando-se em consideração as diferenças por sexo, percebe-se que os adolescentes do sexo masculino, em contexto de acolhimento, apresentam maiores médias, quando comparados às participantes do sexo feminino de ambos os grupos, e também em relação aos adolescentes do mesmo sexo no contexto familiar. 
Anelise Lopes Rodrigues, Lara Lages Gava, Jorge Castellá Sarriera, Débora Dalbosco Dell'Aglio Percepção de preconceito e autoestima entre adolescentes em contexto familiar e em situação de acolhimento institucional

Tabela 2

Resultados da ANOVA. Teste UNIVARIADO

\begin{tabular}{ccccccc}
\hline & Variáveis & Soma dos & & Média & & \\
& Dependentes & quadrados & gl & quadrada & F & Sig. \\
\hline Grupo & Preconceito & $\mathbf{4 0 8 , 5 5 6}$ & $\mathbf{1}$ & $\mathbf{4 0 8 , 5 5 6}$ & $\mathbf{1 6 , 3 2 6}$ & $\mathbf{0 , 0 0 1}$ \\
& Autoestima & 44,601 & 1 & 44,601 & 0,887 & 0,347 \\
\hline Idade & Preconceito & 153,952 & 5 & 30,790 & 1,230 & 0,293 \\
& Autoestima & 255,251 & 5 & 51,050 & 1,016 & 0,407 \\
\hline Sexo & Preconceito & 21,000 & 1 & 21,000 & 0,839 & 0,360 \\
& Autoestima & $\mathbf{4 2 7 , 7 1 8}$ & $\mathbf{1}$ & $\mathbf{4 2 7 , 7 1 8}$ & $\mathbf{8 , 5 1 0}$ & $\mathbf{0 , 0 0 4}$ \\
\hline Grupo* idade & Preconceito & 260,603 & 5 & 52,121 & 2,083 & 0,066 \\
& Autoestima & 286,521 & 5 & 57,304 & 1,140 & 0,338 \\
\hline Grupo* sexo & Preconceito & 8,227 & 1 & 8,227 & 0,329 & 0,567 \\
& Autoestima & $\mathbf{3 4 1 , 3 2 3}$ & $\mathbf{1}$ & $\mathbf{3 4 1 , 3 2 3}$ & $\mathbf{6 , 7 9 1}$ & $\mathbf{0 , 0 0 9}$ \\
\hline
\end{tabular}

A Tabela 2 mostra os resultados da análise univariada de variância (ANOVA). Observa-se diferença significativa na variável preconceito em relação ao grupo $(p<0,001)$ e na variável autoestima em relação ao sexo $(p=0,004)$. Observa-se também diferença significativa na interação grupo*sexo $(p=0,009)$ para a variável autoestima, diferença essa explicada pela média menor das meninas de G2 (contexto de acolhimento institucional) em comparação aos meninos do mesmo grupo.
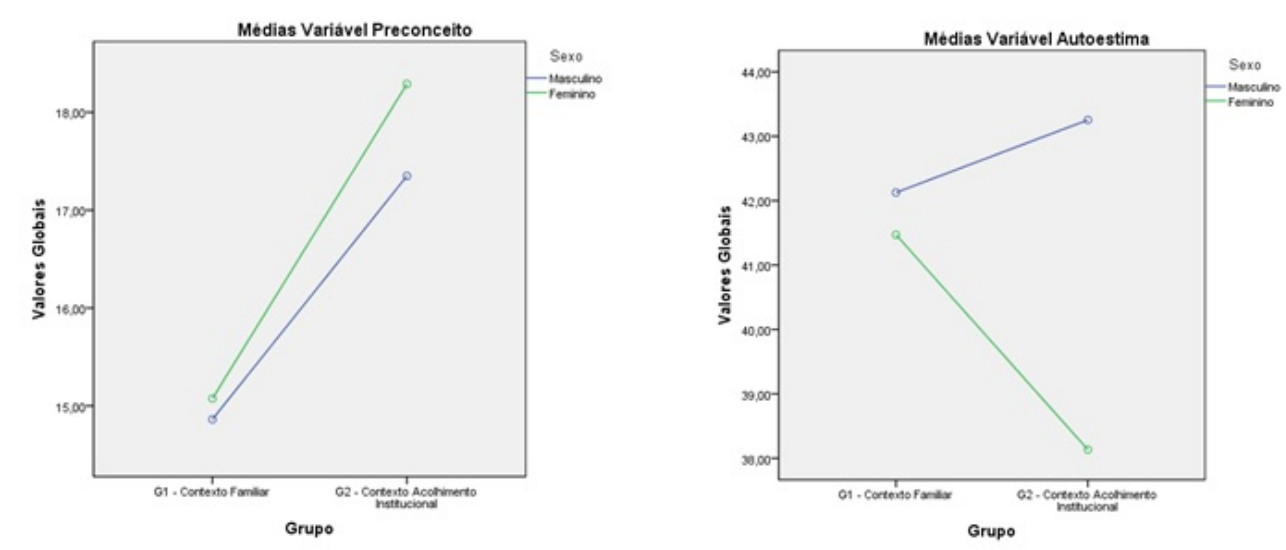

Figura 1. Gráficos de diferença de médias de preconceito e autoestima, por grupo e por sexo 
A Figura 1 apresenta dois gráficos com os valores das médias das variáveis preconceito e autoestima, considerando o grupo e o sexo dos participantes. Observa-se, com relação à percepção de preconceito, que participantes do G2, de ambos os sexos, apresentaram médias bastante elevadas quando comparadas aos participantes do G1. Ao observar os resultados por sexo, desperta atenção ainda que as participantes do sexo feminino tenham apresentado as médias mais elevadas com relação à percepção de preconceito e, ao mesmo tempo, as médias mais baixas com relação à autoestima, comparando-se ambos os grupos.

\section{Discussão}

Os resultados desse estudo sugerem que a situação de acolhimento institucional contribuiu para os índices mais elevados no que se refere à percepção de preconceito por parte dos adolescentes do G2, quando comparados aos do Gl. A situação de acolhimento institucional mostra-se, assim, fortemente marcada por processo de estigmatização social. A literatura anteriormente apresentada aponta que as interações nas instituições de acolhimento, ao serem constituídas em uma matriz sócio-histórica (Rosseti-Ferreira, Amorim, \& Silva, 2004), são perpassadas pelas diferentes significações atribuídas aos institucionalizados, incluindo aí o estigma de fracasso (Arpini, 2003; Siqueira \& Dell' Aglio, 2006). Sendo assim, o fato dos adolescentes em situação de acolhimento apresentarem média significativamente mais elevada na percepção de preconceito pode estar relacionado à própria condição de institucionalização, que Ihes confere uma identidade social, marca-os como diferentes perante a sociedade e os torna, potencialmente, alvos de preconceito. Especificamente no tocante à adolescência, conforme já referido por Arpini (2003), o jovem que vive em uma instituição de acolhimento carrega um forte estigma social, relacionado à crença de que possuem algum tipo de problema ou desvio de conduta. Percebe-se que esse tipo de estigma pode, em algumas situações, levar a efetivas ações de discriminação. A exemplo disso, no estudo realizado por Buffa et al. (2010), os institucionalizados que compuseram a amostra referiram sentir-se excluídos dos demais colegas no contexto da escola. Da mesma forma, os profissionais da instituição de acolhimento entrevistados no estudo relacionaram o sentimento de exclusão referido pelos adolescentes diretamente ao fato dos mesmos estarem em situação de acolhimento.

Em relação à autoestima, embora não tenha havido diferença significativa entre os grupos, esta foi evidenciada entre os sexos, com os meninos apresentando autoestima mais elevada que as meninas. Enquanto pesquisas internacionais mostram que não há um consenso 
em relação à diferença de sexo quanto à autoestima (Sbicigo, Bandeira, \& Dell'Aglio, 2011), o resultado deste estudo vai ao encontro de pesquisas nacionais que apontam maior autoestima entre meninos, sobretudo na adolescência (Romano, Negreiros, \& Martins, 2007; Santos \& Maia, 2003). Essas divergências de resultados nos contextos nacional e internacional sugerem que as diferenças de gênero em autoestima devem ser analisadas contextualmente e, portanto, tratadas com cautela (Hutz \& Zanon, 2011).

Pode-se observar, portanto, que uma maior percepção de preconceito por parte dos adolescentes em situação de acolhimento não se refletiu automaticamente num decréscimo em termos de autoestima, uma vez que não houve diferenças significativas entre os grupos para essa variável. Não obstante, as médias mais elevadas em autoestima dos adolescentes do sexo masculino em contexto de institucionalização, quando comparados aos adolescentes de ambos os sexos do G1, exigem uma maior investigação. Pode-se levantar a hipótese de que as meninas institucionalizadas sejam mais impactadas pela percepção do preconceito, diminuindo sua autoestima, ou ainda, a hipótese de que os meninos de G2 apresentem maior autoestima como forma de superação, talvez por uma estratégia de identificação grupal, conforme referem Pascoe e Richman (2009).

A inconclusividade evidenciada nesse estudo, no que tange às formas como a percepção de preconceito afetam a autoestima de sujeitos pertencentes a grupos estigmatizados, é corroborada pelas divergências teóricas e empíricas, resultantes de diferentes estudos discutidos anteriormente. As previsões das teorias clássicas, apresentadas por Crocker e Major (1989), de que grupos estigmatizados tendem a internalizar a visão negativa atribuída por parte da sociedade e, por consequência, apresentar menor autoestima quando comparados a membros de grupos não estigmatizados, podem ser refutadas a partir de resultados de estudos como os de Bourguignon et al., (2006). Esses autores verificaram que a discriminação voltada para o grupo foi associada positivamente com a autoestima. Desse modo, concluíram que a identificação grupal atuou como moderadora entre a discriminação pessoal e autoestima dos membros de grupos estigmatizados, participantes da amostra, na medida em que auxiliou tais sujeitos a sentirem-se menos sós na sua situação, aliviando assim os maus efeitos gerados pela percepção de preconceito e exclusão.

Em face dos resultados observados neste estudo, é possível considerar que a variável percepção de preconceito mostra-se relacionada à variável identidade grupal, na medida em que os adolescentes em situação de acolhimento investigados percebem-se como parte de um grupo estigmatizado (evidenciado pelos índices mais elevados de preconceito). Por outro lado, tendo em vista que 
não houve diferença na autoestima entre os grupos, é possível considerar que o sentimento de pertença ao grupo possa estar atuando como fator moderador em termos de preservação da autoestima, uma vez que os adolescentes institucionalizados apresentaram médias semelhantes às dos adolescentes que vivem com seus pais.

Torna-se necessário igualmente ressaltar a importância dos fatores situacionais e individuais, que também podem apresentar uma função protetora frente à percepção de discriminação (Pascoe \& Richman, 2009). Desse modo, pode-se compreender que fatores de proteção de caráter situacional, como o apoio social recebido nas casas de acolhimento, e de caráter individual, como estilos de enfrentamento ativo, podem estar mediando o impacto do preconceito percebido sobre a autoestima dos adolescentes institucionalizados que compuseram a amostra.

Por fim, reiteramos que, conforme postulado pelo modelo, os indivíduos não respondem de modo uniforme ao fato de perceberemse alvo de preconceito. É preciso considerar, portanto, uma variabilidade tanto no que diz respeito à percepção de discriminação, quanto no que se refere às consequências dessa percepção em termos de autoestima de membros de grupos estigmatizados (Major et al., 2003).

\section{Considerações finais}

Muito embora os membros de grupos estigmatizados venham sendo retratados tradicionalmente como vítimas passivas dos estereótipos negativos, preconceitos e comportamentos discriminatórios, atribuídos por parte da sociedade (Crocker \& Major, 1989), os resultados deste estudo, alinhados aos de estudos atuais, demonstram que fatores de proteção podem atuar de modo significativo no enfrentamento de situações adversas, como o caso da percepção de preconceito e estigmatização.

Considerando esses resultados e retomando as ideias apresentadas por Arpini (2003), evidencia-se a necessidade de problematizar a tradicional concepção de que as instituições de acolhimento são entidades fracassadas, uma vez que, a despeito de uma maior percepção de preconceito, os adolescentes em situação de abrigamento não apresentaram autoestima significativamente mais baixa que os adolescentes que vivem com seus pais. Tal evidência pode ser considerada um indicador de que as instituições de acolhimento possam representar - para a amostra estudada - um espaço de proteção capaz de fornecer, ainda que provisoriamente, condições para um desenvolvimento saudável. Torna-se, portanto, urgente e necessário repensá-las enquanto espaços de proteção e de 
promoção à saúde de crianças e adolescentes, assim como tornar efetivos os pressupostos apresentados no ECA (1990) e na Lei 12010 (2009), especialmente a questão da convivência familiar e comunitária, de forma que estes adolescentes possam ser percebidos na sua comunidade como os demais adolescentes.

Para estudos futuros, sugere-se a realização de pesquisas de caráter contextual e com abordagens qualitativas capazes de melhor explorar as relações entre a percepção de preconceito e a autoestima em adolescentes em situação de institucionalização. Além disso, são recomendados estudos longitudinais, que possam observar a variação da autoestima e da percepção de preconceito em adolescentes que ingressam no sistema de acolhimento institucional ou que são reinseridos em suas famílias, de forma que possa ser mais bem avaliado o impacto da institucionalização em seu bem-estar. Mais especialmente, sugere-se que a questão de gênero possa ser melhor investigada, especialmente no que se refere à autoestima em meninas institucionalizadas, já que estudos anteriores indicaram maior manifestação de sintomas depressivos neste grupo (Dell'Aglio, Borges, \& Santos, 2004; Wathier \& Dell'Aglio, 2007; Dell'Aglio \& Hutz, 2004), o que também pode estar relacionado a uma menor autoestima. Estudos sobre estas questões permitem compreender em profundidade - as peculiaridades do desenvolvimento em situação de institucionalização e podem viabilizar a identificação das fontes de vulnerabilidade e também dos fatores promotores de proteção que possam efetivamente auxiliar esses adolescentes a fazer frente às situações de preconceito, estigmatização e discriminação vivenciadas em sociedade.

\section{Referências}

Allport, G. W. (1954/1979). The nature of prejudice. Reading, MA: Addison-Wesley.

Arpini, D. M. (2003). Repensando a perspectiva institucional e a intervenção em abrigos para crianças e adolescentes. Psicologia Ciência e Profissão, 21(3), 70-75.

Ayres, L. S., Coutinho, A. P., Sá, D. A., \& Albernaz, T.(2010). Abrigo e abrigados: Construções e desconstruções de um estigma. Estudos e Pesquisas em Psicologia, 10(2), 420-433.

Barbetta, P. A. (2001). Estatística aplicada às ciências sociais. 4 ed. Florianópolis: UFSC.

Bianchi, F. T., Zea, M. C., Belgrave, F. Z., \& Echverry, J. J. (2002). Racial Identity and self-esteem among black Brazilian men: Race matters in Brazil too! Cultural Diversity and Ethnic Minority Psychology, 8(2), 157-169. 
Bourguignon, D., Seron, E., Yzerbyt, V., \& Herman, G. (2006). Perceived group and personal discrimination: Differential effects on personal self-esteem. European Journal of Social Psychology, 36, 773-789.

Buffa, C. G, Teixeira, S. C., \& Rosseti-Ferreira, M. C. (2010). Vivências de exclusão em crianças abrigadas. Psicologia: Teoria e Prática, 12(2), 17-34.

Casas, F. (2010). El bienestar personal: Su investigación en la infancia y la adolescencia. Encuentros en Psicología Social, 5(1), 85-101.

Clam - Centro Latino Americano em Sexualidade e Direitos Humanos -I MS/UERJ (2009). Gênero e diversidade na escola: Formação de professores em gênero, orientação sexual e relações étnicoraciais. Livro de conteúdo. Rio de Janeiro: CEPESC; Brasília: SPM.

Crocker, J., \& Major, B. (1989). Social stigma and self-esteem: The self protective properties of stigma. Psychological Review, 96, 608-630.

Crocker, J., \& Quinn, D. M. (2000). Social stigma and the self: Meanings, situations, and self-esteem. In T. F. Heatherton, \& R. E. Kleck (Eds.), The social psychology of stigma (pp. 153-183). New York: Guilford Press.

Dell'Aglio, D. D., Borges, J. L., \& Santos, S. S. (2004). Eventos estressores e depressão em adolescentes do sexo feminino. Psico, 35(1), 43-50.

Dell'Aglio, D. D., \& Hutz, C. D. (2004). Depressão e desempenho escolar em crianças e adolescentes institucionalizados. Psicologia: Reflexão e Crítica, 17(3), 351-357.

Dell'Aglio, D. D., Koller, S. H., Cerqueira-Santos, E., \& Colaço, V. F. R. (2011). Revisando o Questionário da Juventude Brasileira: Uma nova proposta. In D. D. Dell'Aglio, \& S. H. Koller (Orgs.), Adolescência e Juventude: vulnerabilidade e contextos de proteção (pp. 259-270). São Paulo: Casa do Psicólogo.

Diener, E. (1984). Subjective well-being. Psychological Bulletin, 95(3), 542-575.

Estatuto da Criança e do Adolescente. (1990). Lei Federal no 8069, de 13 de julho de 1990. Brasília, DF: Presidência da República.

Hutz, C. S., \& Zanon, C. (2011). Revisão da adaptação, validação e normatização da Escala de Autoestima de Rosenberg. Avaliação Psicológica, 10(1), 41-49.

Lei no 12.010, de 3 de agosto de 2009. (2009, 4 de agosto). Dispõe sobre adoção; altera as Leis nos 8.069, de 13 de julho de 1990 Estatuto da Criança e do Adolescente, 8.560, de 29 de dezembro de 1992; revoga dispositivos da Lei $\mathrm{n}^{\circ}$ 10.406, de 10 de janeiro de 2002 - Código Civil, e da Consolidação das Leis do Trabalho - CLT, aprovada pelo Decreto-Lei $\mathrm{n}^{\circ} 5.452$, de $1^{\circ}$ ㅇ 
maio de 1943; e dá outras providências. Brasília, DF. Recuperado em 07 outubro, 2011, de http://www.planalto.gov.br/ccivil_03/_Ato2007/2010/2009/Lei/ L12010.htm

Major, B., Quinton, W. J., \& Mccoy, S. K. (2002). Antecedents and consequences of attributions to discrimination: Theoretical and empirical advances. In M. P. Zanna (Ed.), Advances in experimental social psychology (Vol.34, pp. 251-330). San Diego: Academic Press.

Major, B., McCoy, S. K., Kaiser, C.R., \& Quinton, W.J. (2003). Prejudice and self esteem: A transactional model. European Review of Social Psychology, 14, 77-104.

Major, B., \& O'brien, L. T. (2005). The social psychology of stigma. Annual Review of Psychology, 56, 393-421.

Ministério da Saúde. Conselho Nacional de Saúde. (2002). Resolução № 196 de 10 de outubro de 1996. In Manual operacional para comitês de ética em pesquisa (pp.83-100). Brasília: Ministério da Saúde.

Nunes, J. G., Santos, M., Martins, I. N., \& Monteiro, C. A. (2010). O cotidiano de crianças e adolescentes abrigadas no oratório festivo São João Bosco - Oratório de Bebé. Cadernos de Graduação - Ciências Humanas e Sociais, 12(12), 183-198.

Pascoe, E. A., \& Richman, L. S. (2009). Perceived discrimination and health: A metaanalytic review. Psychological Bulletin, 135, 531554.

Prada, C., Williams, L., \& Weber, L. (2007). Abrigos para crianças vítimas de violência doméstica: Funcionamento relatado pelas crianças e pelos dirigentes. Psicologia: Teoria e Prática, 9(2), $14-25$.

Rizzini, I. (2006). Acolhendo crianças e adolescentes: Experiências de promoção do direito à convivência familiar e comunitária no Brasil. São Paulo: Cortez.

Romano, A., Negreiros, J., \& Martins, T. (2007). Contributos para validação da Escala de Autoestima de Rosenberg numa amostra de adolescentes da região interior norte do país. Psicologia, Saúde \& Doenças, 8(1), 109-116.

Rosenberg, M. (1965). Society and the adolescent self-image. Princeton: Princeton University Press.

Rosseti-Ferreira, M. C., Amorin, K. S., \& Silva, A. P. S. (2004). Rede de significações: Alguns conceitos básicos. In M. C. RossetiFerreira, K. S. Amorin, \& A. P. S. Silva (Orgs.), Rede de significações e o estudo do desenvolvimento humano ( $p p$. 23-33). Porto Alegre: Artmed. 
Sbicigo, J. B., Bandeira, D. R., \& Dell'Aglio, D. D. (2011). Escala de Autoestima de Rosenberg (EAR): Validade fatorial e consistência interna. Psico-USF, 15, 395-403.

Sidanius, J., \& Pratto, F. (1999). Social dominance: An intergroup Theory of Social Hierarchy and Opression. Cambridge: Cambridge University Press.

Silva, E. R. (2004). O direito à convivência familiar e comunitária: Os abrigos para crianças e adolescentes no Brasil. Brasília: I pea/Conanda.

Santos, P. J., \& Maia, J. (2003). Análise factorial confirmatória e validação preliminar de uma versão portuguesa da escala de auto-estima de Rosenberg. Psicologia: Teoria, Investigação e Prática, 2, 253-268.

Siqueira, A. C., \& Dell’Aglio, D. D. (2006). O impacto da institucionalização na infância e adolescência: Uma revisão de literatura. Psicologia \& Sociedade, 18(1), 71-80.

Siqueira, A. C., Zoltowski, A. P., Giordani, J. , Otero, T., \& Dell'Aglio, D. D. (2010) Processo de reinserção familiar: Estudo de casos de adolescentes que viveram em instituição de abrigo. Estudos de Psicologia, 15(1), 7-15.

Twenge, J. M., \& Crocker, J. (2002). Race and self-esteem: Metaanalysis comparing Whites, Blacks, Hispanics, Asians, and American Indians and comment on Gray-Little and Hafdah. Psychological Bulletin, 128, 371-408.

Wathier, J. L., \& Dell'Aglio, D. D.(2007). Sintomas depressivos e eventos estressores em crianças e adolescentes no contexto de institucionalização. Revista de Psiquiatria do Rio Grande do Sul, 29, 305-314.

\section{Endereço para correspondência \\ Anelise Lopes Rodrigues}

Universidade Federal do Rio Grande do Sul

Rua Ramiro Barcelos, 2600/122, CEP 90035-003, Porto Alegre - RS, Brasil

Endereço eletrônico: aneliselr@hotmail.com

\section{Lara Lages Gava}

Universidade Federal do Rio Grande do Sul

Rua Ramiro Barcelos, 2600/115, CEP 90035-003, Porto Alegre - RS, Brasil

Endereço eletrônico: laralagesgava@gmail.com

\section{Jorge Castellá Sarriera}

Universidade Federal do Rio Grande do Sul

Rua Ramiro Barcelos, 2600/122, CEP 90035-003, Porto Alegre - RS, Brasil

Endereço eletrônico: jorgesarriera@gmail.com

\section{Débora Dalbosco Dell'Aglio}

Universidade Federal do Rio Grande do Sul

Rua Ramiro Barcelos, 2600/115, CEP 90035-003, Porto Alegre - RS, Brasil

Endereço eletrônico: dalbosco@cpovo.net

Recebido em: 26/03/2012

Reformulado em: 24/01/2014 
Anelise Lopes Rodrigues, Lara Lages Gava, Jorge Castellá Sarriera, Débora Dalbosco Dell'Aglio Percepção de preconceito e autoestima entre adolescentes em contexto familiar e em situação de acolhimento institucional

Aceito para publicação em: 28/01/2014

\section{Notas}

* Psicóloga, Doutora em Psicologia pela Universidade Federal do Rio Grande do Sul (Bolsista CNPq - Brasil); Mestre em Psicologia Social e da Personalidade pela Pontifícia Universidade Católica do Rio Grande do Sul, Porto Alegre / RS, Brasil.

** Psicóloga, Mestre em Filosofia e Doutora em Psicologia pela Universidade Federal do Rio Grande do Sul, Porto Alegre/RS, Brasil.

*** Doutor em Psicologia Social pela Universidade Autônoma de Madrid; Professor no Programa de Pós-Graduação em Psicologia da Universidade Federal do Rio Grande do Sul, Porto Alegre/RS, Brasil; Bolsista Produtividade CNPq; Coordenador do Grupo de Pesquisa em Psicologia Comunitária.

**** Psicóloga, Doutora em Psicologia do Desenvolvimento, Docente do Programa de Pós-Graduação em Psicologia da Universidade Federal do Rio Grande do Sul, Porto Alegre/RS, Brasil. 